\title{
A torsional model of multi-stage gears - influence of external excitations and tooth shape modifications
}

\author{
Hassen Fakhfakh ${ }^{1}$, Jérôme Bruyère ${ }^{1}$, Philippe Velex ${ }^{1, a}$ and Samuel Becquerelle ${ }^{2}$ \\ 1 INSA Lyon, LaMCoS, 18-20 rue des Sciences, 69100 Villeurbanne, France \\ 2 Hispano-Suiza, 18 Boulevard Louis Seguin, 92700 Colombes, France
}

Received 18 May 2015, Accepted 6 November 2015

\begin{abstract}
In this paper, a torsional dynamic model of multi-stage idler spur and helical gears is presented which combines mesh internal excitations and external forcing terms such as time-varying external torque. Each contact line in the various base planes is discretized in elemental cells which are all attributed a time-varying mesh stiffness element and initial separation to account for tooth shape deviations from ideal involute flanks. The mesh stiffness functions are estimated from the formulae of Weber \& Banaschek and their relative phasing is determined based on gear geometry and relative positioning. The corresponding non-linear differential system is solved by combining a Newmark's numerical time-step integration scheme and a normal contact algorithm. A number of simulation results are presented on the combined influence on dynamic tooth loads of errors and shape deviations along with external excitations.
\end{abstract}

Key words: Spur and helical gears / gear dynamics / multi-stage gears / tooth shape deviations

\section{Introduction}

In a number of mechanisms, multi-stage geared systems need to be employed to reduce speed and/or split the total power into several paths in order to operate pumps, generators, motors, etc. In such conditions, load machines control the load sharing between the various meshes to a large extent and can also introduce additional excitations via time-varying resisting torque for example. It is therefore interesting to analyse the potential influence of power circulation on the dynamic behaviour of multi-stage geared systems along with the contributions of mesh and external excitations. Most of the literature on gear dynamics deals with individual pinion-gear pairs and only a limited number of papers on multi-stage gears can be found. Choy et al. [1,2] investigated the modal response of double-stage gears for steady-state and transient conditions. Velex and Saada [3], Velex and Raclot [4], Kubur et al. [5] introduced finite element models of two-stage units and investigated the influence of key design parameters on the system dynamics. More recently, Carbonelli et al. $[6,7]$ presented a robust optimization method for tooth corrections in truck timing gear cascades based on a swarm particle algorithm to minimize static transmission error time-variations. $\mathrm{Yu}$ et al. [8] proposed a dynamic

\footnotetext{
${ }^{a}$ Corresponding author:

Philippe.Velex@insa-lyon.fr
}

model with several pinions and studied the contribution of mesh frequency, bearing stiffness to the load sharing properties. Yang [9] studied the vibrational response in torsion of a two-stage gear submitted to deterministic and random excitations in the presence of backlash.

The objective of this paper is to present a simplified torsional model that can simulate dynamic tooth loads on several idler gears in the presence of time-varying mesh stiffness functions and tooth shape modifications. The main contribution is the analysis of the influence of power circulation when several constant or time-varying torques are applied which have been rarely tackled in the literature. Unlike the majority of the models, transmission errors are one of the results of the simulations and dynamic responses are derived by the simultaneous solution of the instant contact conditions on the teeth and the equations of motion.

\section{Theory}

\subsection{Model of $\mathrm{N}-1$ stage gear systems}

The model is composed of $\mathrm{N}$ idler spur or helical pinions simulated as rigid discs and connected by time-varying mesh stiffness functions [5, 10] (Fig. 1). Each member has one single torsional degree-of-freedom $\left(\theta_{1}, \theta_{2}, \ldots, \theta_{N}\right)$ which represents the angular perturbation 


\section{Nomenclature}

\begin{tabular}{|c|c|}
\hline$\vec{R}_{m}\left(\vec{X}_{m}, \vec{Y}_{m}, \vec{Z}\right)$ & Reference linked to the base plane of stage $m$ \\
\hline$a, b$ & Rayleigh damping constants (here $a=1,12 ; b=1,2 \times 10^{-5}$ ) \\
\hline$A_{1}, A_{2}, B_{1}, B_{2}, C_{1}, C_{2}, C_{3}, M$ & Geometrical points defined in Figures 2 and 3 \\
\hline$C t_{i}$ & Input/ output torque of pinion $i$ (N.m) \\
\hline$C_{0}, C_{s}$ & Nominal torque, peak value of the periodic signal (N.m) \\
\hline$\Omega_{i}$ & Rigid body angular speed of pinion $i\left(\operatorname{rad} . \mathrm{s}^{-1}\right)$ \\
\hline$O_{i}$ & Centre of pinion $i$ \\
\hline$\theta_{i}$ & Torsional degree-of-freedom of pinion $i$ (rad) \\
\hline$\gamma_{i}$ & $\begin{array}{l}\text { Angle between the axis of pinions } i \text { and } i+1 \\
\text { (positively defined in the trigonometric sense) (rad) }\end{array}$ \\
\hline$z_{i}$ & Tooth number of pinion $i$ \\
\hline$\alpha_{t i}$ & Apparent pressure angle of pinion $i(\mathrm{rad})$ \\
\hline$R b_{i}$ & Base radius of pinion $i(\mathrm{~m})$ \\
\hline$J_{i}$ & Polar moment of inertia of pinion $i\left(\mathrm{~kg} \cdot \mathrm{m}^{2}\right)$ \\
\hline$S b a, P b a$ & Apparent base tooth thickness, base pitch (m) \\
\hline$\beta_{b}$ & Base helix angle (rad) \\
\hline$M_{m}$ & Potential point of contact \\
\hline$e_{m}\left(M_{m}\right)$ & Composit deviation at $M_{m}$ at the initial state before deformation (m) \\
\hline$\delta e_{m}\left(M_{m}\right)$ & $=\max \left(e_{m}\left(M_{m}\right)\right)-e_{m}\left(M_{m}\right)(\mathrm{m})$ \\
\hline$\delta_{m}\left(M_{m}\right)$ & Normal approach at $M_{m}$ from rigid body condition (m) \\
\hline$E_{\max _{m}}(t)$ & $=\max \left(e_{m}\left(M_{m}\right)\right)(\mathrm{m})$ \\
\hline$\varepsilon_{\alpha_{m}}$ & Contact ratio of stage $m$ \\
\hline$\varepsilon_{1}$ & Sign of $\Omega_{1}$ \\
\hline$\zeta_{M_{m}}$ & Sign of torque $C t_{m}$ \\
\hline$f_{t} \cdot \Omega$ & Periodic torque frequency $\left(\mathrm{rad} . \mathrm{s}^{-1}\right)$ \\
\hline$t$ & Time (s) \\
\hline$T_{s y s}, T_{m}$ & System period, mesh period $(\mathrm{s})$ \\
\hline LCM & Least common multiple \\
\hline$k_{h}$ & Additional torsional stiffness placed at node $N\left(\right.$ N.m.rad $\left.{ }^{-1}\right)$ \\
\hline$k_{m}\left(M_{m}\right)$ & Mesh stiffness associated with the discrete cell centered at $M_{m}$ of stage $m\left(\mathrm{~N} . \mathrm{m}^{-1}\right)$ \\
\hline$\left[K_{a v}\right]$ & Average stiffness matrix \\
\hline$T m_{1}, T m_{2}$ & Limits of base plane for stage $m$ \\
\hline$T^{\prime} m_{1}, T^{\prime} m_{2}$ & Limits of meshing area on the base plane of stage $m$ \\
\hline $\mathbf{V}_{\mathbf{m}}$ & Structural vector associated with stage $m$ \\
\hline$\Delta l$ & Phase difference measured on base plane \\
\hline$V_{b}$ & Linear speed $\left(\mathrm{m} . \mathrm{s}^{-1}\right)$ \\
\hline$\Phi_{c}, \phi$ & Angles between two lines of action (see Figs. 2 and 3) (rad) \\
\hline\|\| & Remainder of the natural division \\
\hline$\overparen{A B}$ & Arc length between points A and B \\
\hline
\end{tabular}

superimposed on rigid-body rotation. All the contact lines in the base planes are discretized into thin slices which are all attributed (i) an elemental time-varying mesh stiffness, and (ii) a normal deviation to account for mesh stiffness variations (including their relative phasing) and tooth shape deviations with respect to perfect involute tooth flanks. Constant or time-varying external loads can be introduced at any node of the model and, in this paper, periodic torques of the form $C t(t)=C_{0}+C_{s} \sin \left(f_{t} \Omega t\right)$ will be considered where $\Omega$ is the rotational frequency of the member.

\subsection{Rigid body motions - Tooth shape modifications}

For multi-stage idler gears, every mesh can alter the speed ratio because of its individual errors and shape deviations. Rigid-body kinematics can be characterised by extending the formulation in reference [11] to system comprising $N$ meshes which leads to the following expression for the $i$ th member angular speed:

$$
\Omega_{i}(t)=(-1)^{i-1}\left[\frac{R b_{1}}{R b_{i}} \Omega_{1}(t)+\varepsilon_{1} \frac{\mathrm{d}}{\mathrm{d} t}\left(\frac{\sum_{m=1}^{i-1} E_{\max _{m}}(t)}{R b_{i} \cos \beta_{b}}\right)\right]
$$

Tooth shape modifications are accounted for by distributions of equivalent normal deviations with respect to the ideal flanks for both the pinion and the gear. At any potential point of contact in the base plane, the corresponding initial gaps (before deformation) are updated based on the relative rigid motion of the teeth. By adding 


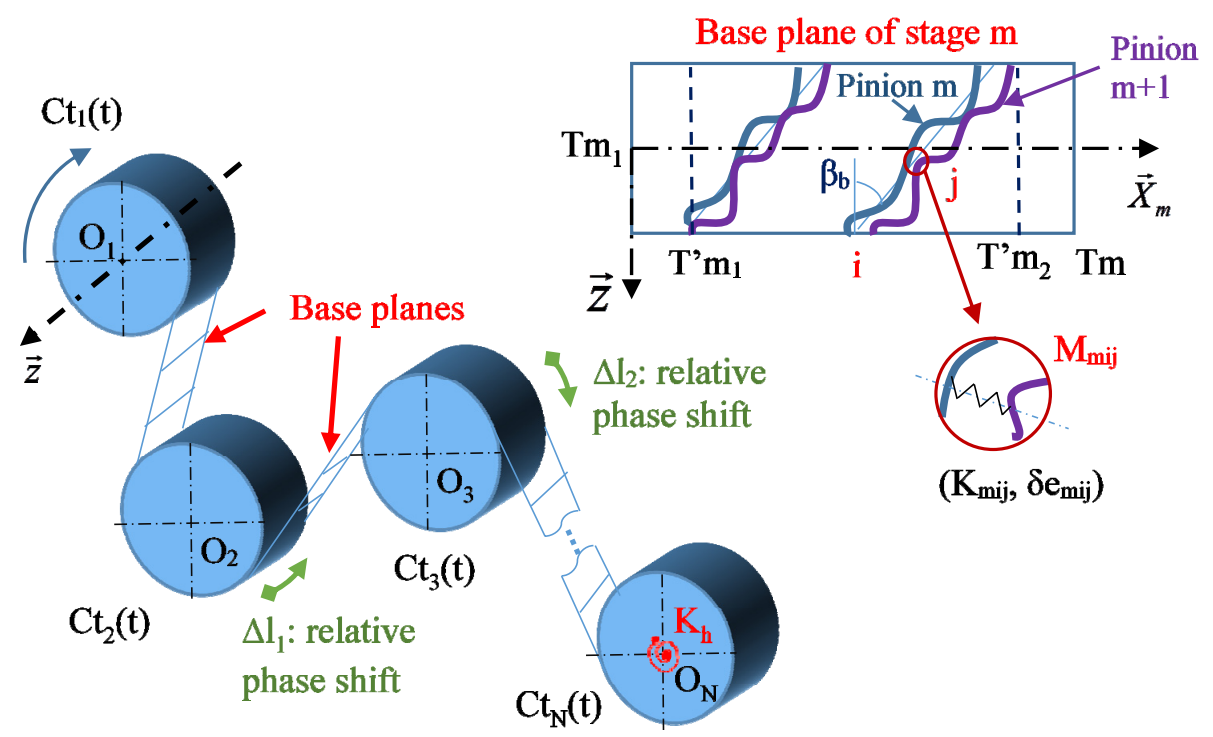

Fig. 1. Multi-stage idler gear model.

the contributions from the mating pinion and gear flanks, initial separations $\delta e_{m}\left(M_{m}\right)$ are defined for each mesh whose relative time-phasing is determined as explained in the following section.

\subsection{Mesh phasing}

Mesh phasing in multi-stage idler gears is a key parameter since it controls the combinations of mesh excitations in terms of mesh stiffness functions and tooth shape modifications. Two general situations can be identified depending on the position of the power input and the angular positions of the pinions. Considering first the case of three members such that the driving gear is either the first or the third one in the kinematic chain (Fig. 2), the meshing conditions on the two base planes are linked and the phase difference on the second base plane (with respect to the first one) can be expressed as a distance $\Delta l$ such that [12]

$$
\Delta l= \begin{cases}S b a-\kappa_{l} P b a & \text { if } \kappa_{l} P b a \leqslant S b a \\ S b a+\left(1-\kappa_{l}\right) P b a & \text { if } \kappa_{l} P b a>S b a\end{cases}
$$

where $\kappa_{l}=\left\|\frac{\left|T^{\prime} 1_{1} T^{\prime} 1_{2}\right|}{P b a}\right\|$ with \|\|$:$ remainder of the natural division.

Distance $\left|T^{\prime} 1_{1} T^{\prime} 1_{2}\right|$ depends on angle $\phi$ (Fig. 2) hence on the relative positions of the three pinions and reads:

$$
\left|T^{\prime} 1_{1} T^{\prime} 1_{2}\right|=\left|T^{\prime} 1_{1} T 2_{1}\right|+\phi R b_{2}+\left|T 1_{2} T^{\prime} 1_{2}\right|
$$

where $\phi$ is the angle between the two lines of actions (dependent on the sign of the driving torque $\left.C t_{1}\right)$ :

$$
\phi=\left|\kappa_{e} 2 \pi-\kappa_{s} \gamma_{1}-\alpha_{t 1}-\alpha_{t 2}\right|
$$

with $\left\{\begin{array}{l}\kappa_{e}=0 \text { and } \kappa_{s}=-1 \text { if } C t_{1}<0 \\ \kappa_{e}=1 \text { and } \kappa_{s}=1 \text { if } C t_{1}>0\end{array}\right.$ $\gamma_{1}$ is the angle between axes $\left(\mathrm{O}_{1} \mathrm{O}_{2}\right)$ and $\left(\mathrm{O}_{2} \mathrm{O}_{3}\right)$ (in the trigonometric sense).

The second case of interest corresponds to three members where the driving pinion is the intermediate one (Fig. 3) for which the phase difference between the two meshes defined as a distance on the base plane becomes:

$$
\Delta l=\left\|\frac{\left|T^{\prime} 1_{2} M\right|}{P b a}\right\|
$$

with $\left|T^{\prime} 1_{2} M\right|$ the distance between the first point of contact $T^{\prime} 1_{2}$ and point $\mathrm{M}$ :

$$
\begin{aligned}
& \left|T^{\prime} 1_{2} M\right|=\widehat{C_{3} C_{2}}=\widehat{A_{1} C_{1}}+\widehat{C_{1} C_{2}}-\widehat{A_{1} A_{2}}-\widehat{A_{2} C_{3}} \\
& \left|T^{\prime} 1_{2} M\right|=\left|A_{1} T^{\prime} 1_{1}\right|+\kappa_{c} n P b a-\kappa_{c} \Phi_{c} R b_{2}-\left|A_{2} T^{\prime} 1_{2}\right|
\end{aligned}
$$

where $\Phi_{c}=\left\{\begin{array}{ll}2 \pi-\gamma_{1}-\alpha_{t 1}+\alpha_{t 2} & \text { if } C t_{2}>0 \\ 2 \pi-\gamma_{1}+\alpha_{t 1}-\alpha_{t 2} & \text { if } C t_{2}<0\end{array}\right.$ and $\kappa_{c}=$ $\left\{\begin{aligned} 1 & \text { if } C t_{2}>0 \\ -1 & \text { if } C t_{2}<0\end{aligned}\right.$ (see Fig. 3 for the parameter definition) $C_{3} C_{2}$ is the length of arc $\mathrm{C}_{3} \mathrm{C}_{2} ; \gamma_{1}$ is the angle between axes $\left(\mathrm{O}_{1} \mathrm{O}_{2}\right)$ and $\left(\mathrm{O}_{2} \mathrm{O}_{3}\right)$ (in the trigonometric sense) and $n P b a$ is an integral multiple of $\mathrm{Pba}$.

\subsection{Equation of motion in the presence of shape deviations/errors}

At any potential point $M_{m}$ on a base plane, the mesh deflection is the normal approach relative to rigid-body positions from which the initial normal separation due to errors or/and shape modifications is subtracted:

$$
\Delta_{m}\left(M_{m}\right)=\delta_{m}\left(M_{m}\right)-\delta e_{m}\left(M_{m}\right)
$$

Based on the gear mesh interface model in Figure 1, and, after applying the resulting total mesh forces and moment 


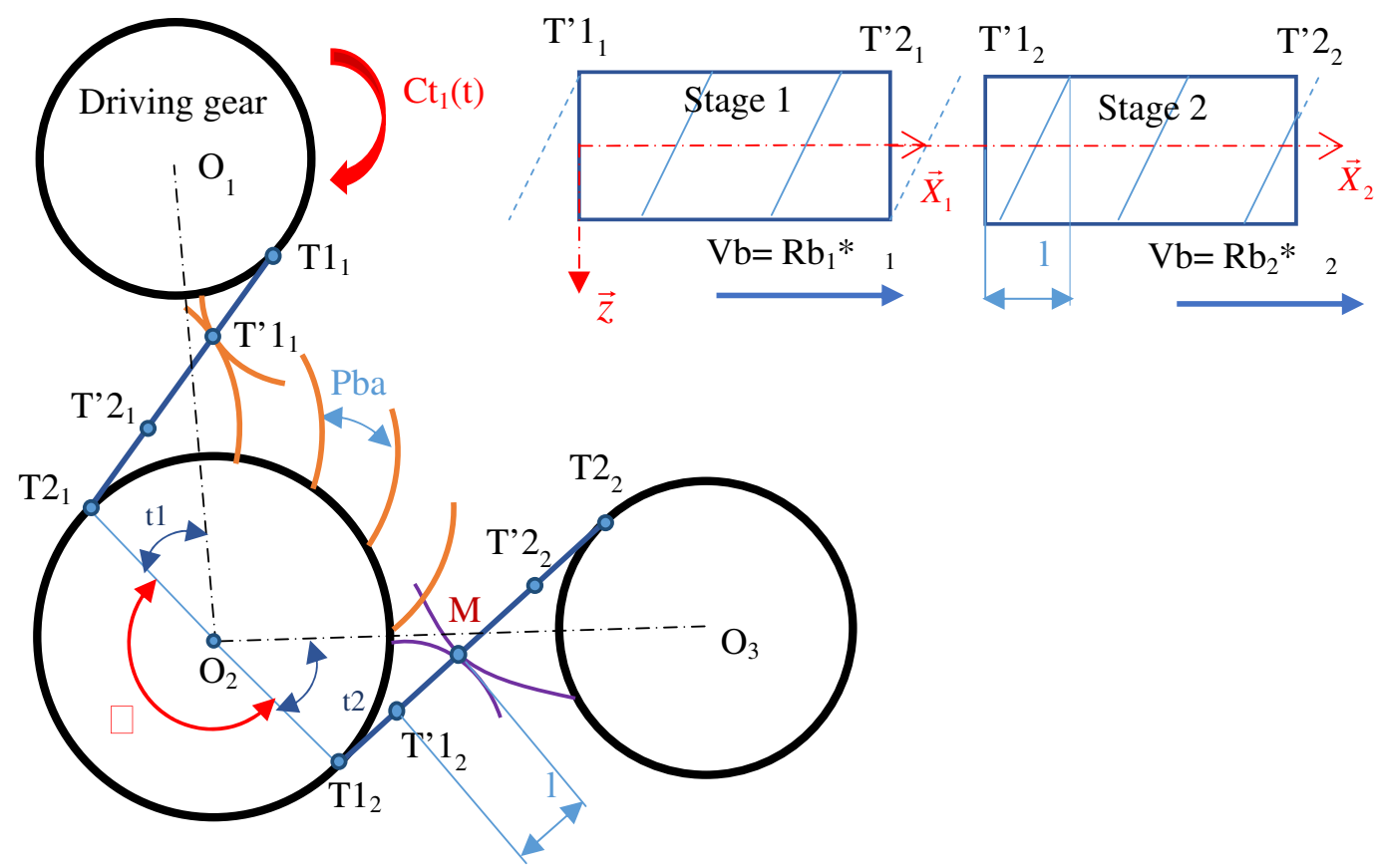

Fig. 2. 1st configuration: 3 members with the driving pinion being either the first or third member.

$$
\mathbf{V}_{\mathbf{m}}=\left\langle\begin{array}{llll}
\underbrace{0 \cdots 0}_{(m-1) \text { terms }} & \zeta_{M m} R b_{m} \cos \beta_{b} & \zeta_{M m} R b_{m+1} \cos \beta_{b} & \underbrace{0 \cdots \cdots}_{(N-m-1) \text { terms }}
\end{array}\right\rangle
$$

at the centres of the pinion and gear of each stage [13], the inter-force wrench associated with mesh $m$ can be deduced in a compact form as:

$$
\mathbf{F}_{\mathbf{M}}=-\left[K_{m}(t)\right] \mathbf{q}+\left\{F_{m}\left(t, \delta e_{m}\left(M_{m}\right)\right)\right\}
$$

where $\left[K_{m}(t)\right]=\int_{L(t, \mathbf{q})} k_{m}\left(M_{m}\right) \mathbf{V}_{\mathbf{m}}\left(M_{m}\right) \mathbf{V}_{\mathbf{m}}\left(M_{m}\right)^{T} \mathrm{~d} M$ is the mesh stiffness matrix, $\left\{F_{m}\left(t, \delta e_{m}\left(M_{m}\right)\right)\right\}=$ $\int k_{m}\left(M_{m}\right) \delta e_{m}\left(M_{m}\right) \mathbf{V}_{\mathbf{m}}\left(M_{m}\right) \mathrm{d} M$ embodies the con$L(t, \mathbf{q})$

tributions of shape deviations and errors, $\mathbf{V}_{\mathbf{m}}$ is a structural vector whose components are defined in the equation above, is a structural vector and $\mathbf{q}=\left\langle\theta_{1}, \theta_{2} \ldots \theta_{N}\right\rangle$ is the degree-of-freedom vector.

Following the same procedure as for the inter-force wrench, the global mass matrix and the global inertial vector term for $\mathrm{N}$ idler torsional geared system is obtain under the form $\mathbf{M}=\operatorname{diag}\left(J_{1}, J_{2}, \ldots J_{N}\right)$ and $\left\{F_{2}(t)\right\}=\left\langle-J_{1} \dot{\Omega}_{1}-J_{2} \dot{\Omega}_{2} \ldots-J_{N} \dot{\Omega}_{N}\right\rangle$. In these conditions, the equations of motion read:

$$
\begin{aligned}
\mathbf{M} \ddot{\mathbf{q}}+\mathbf{C} \dot{\mathbf{q}}+[K(t, \mathbf{q})] \mathbf{q} & =\left\{F_{0}(t)\right\} \\
+ & \left\{F_{1}\left(t, \mathbf{q}, \delta e_{m}\right)\right\}+\left\{F_{2}(t)\right\}
\end{aligned}
$$

where $\mathbf{M}$ and $[K(t, \mathbf{q})]$ are the global mass and stiffness matrices, the damping matrix is expressed using a Rayleigh model such that $\mathbf{C}=a \mathbf{M}+b\left[K_{a v}\right]$ where $\mathrm{a}$ and $\mathrm{b}$ are two constants [14], $\left\{F_{0}(t)\right\}=$ $\left\langle C t_{1}(t) C t_{2}(t) \ldots C t_{N}(t)\right\rangle$ is the external torque vector and $\left\{F_{1}\left(t, \mathbf{q}, \delta e_{m}\right)\right\}$ embodies the contributions of shape deviations and errors.

$$
\text { See equations (10) and (11) next page. }
$$

In what follows, the equations of motion are solved by using a Newmark's time-step integration scheme combined with a normal contact algorithm which verifies that all the contact forces between the tooth flanks are compressive.

\subsection{Applications}

The numerical applications are conducted on the 3stage idler spur gear system defined in Figure 4 and Table 1 , the pinion/gear centres are all aligned such that $\gamma_{1}=\gamma_{2}=180^{\circ}$.

\subsubsection{Oscillating torque}

In a number of applications, the total power is split between several auxiliary components such as pumps, generators, motors, etc., and the influence of the additional intermediate resisting torques on the overall dynamic behaviour needs to be investigated. In this section, four different examples of power circulations are considered: (a) a constant driving torque $C t_{1}=500$ N.m; (b) a driving torque with a periodic component $C t_{1}(t)=\left[500+100 \sin \left(10 \Omega_{1} t\right)\right]$ N.m on pinion 1 and a resisting torque on the output gear 4 (adjusted 


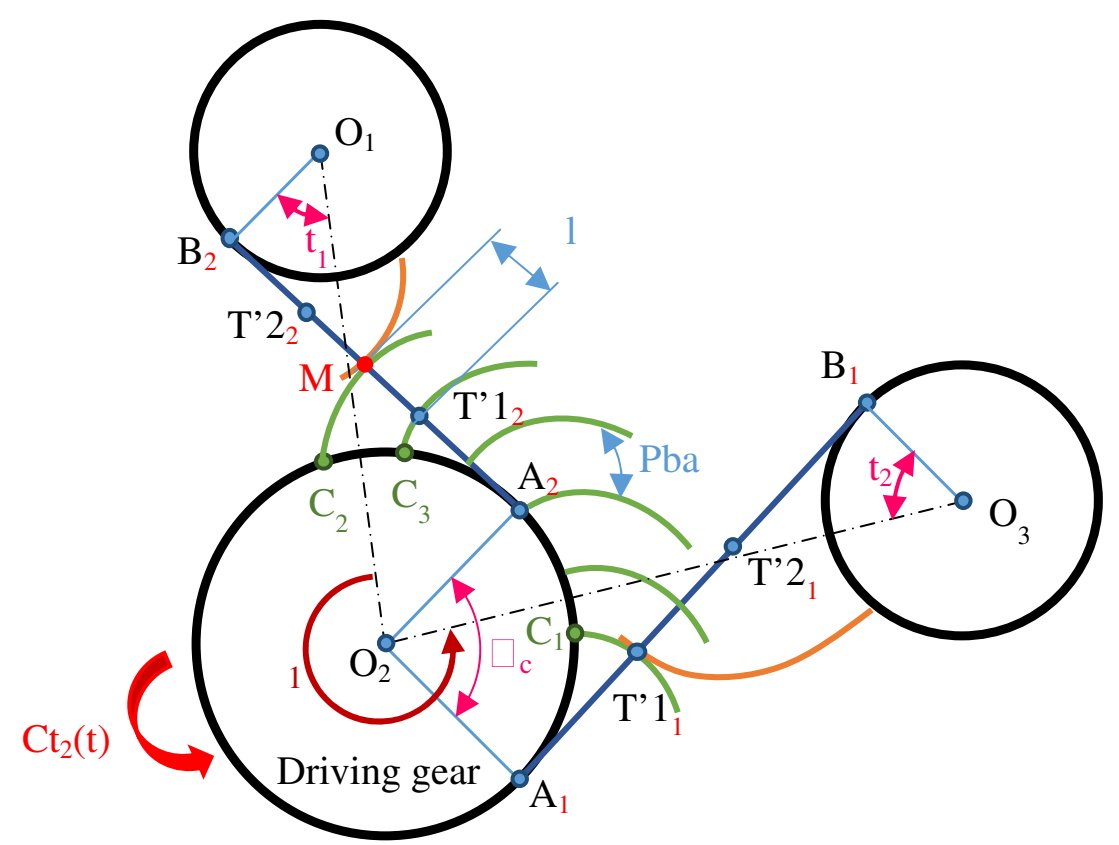

Fig. 3. 2nd configuration: 3 members with the driving pinion being the intermediate member.

$$
[K(t, \mathbf{q})]=\cos ^{2}\left(\beta_{b}\right)\left[\begin{array}{ccccc}
k_{1}(t) R b_{1}^{2} & k_{1}(t) R b_{1} R b_{2} & 0 & 0 & 0 \\
k_{1}(t) R b_{1} R b_{2} & \left(k_{1}(t)+k_{2}(t)\right) R b_{2}^{2} & k_{2}(t) R b_{2} R b_{3} & 0 & 0 \\
0 & k_{2}(t) R b_{2} R b_{3} & \left(k_{2}(t)+k_{3}(t)\right) R b_{3}^{2} & \ldots & 0 \\
0 & 0 & \vdots & & \\
0 & 0 & & & k_{N-1}(t) R b_{N}^{2}+k_{h}
\end{array}\right]
$$

$$
\left\{F_{1}\left(t, \mathbf{q}, \delta e_{m}\right)\right\}=\left\{\begin{array}{c}
k_{1}(t) \delta e_{1}(M) \zeta_{M 1} R b_{1} \cos \beta_{b} \\
\left(k_{1}(t) \delta e_{1}(M) \zeta_{M 1}+k_{2}(t) \delta e_{2}(M) \zeta_{M 2}\right) R b_{2} \cos \beta_{b} \\
\left(k_{2}(t) \delta e_{2}(M) \zeta_{M 2}+k_{3}(t) \delta e_{3}(M) \zeta_{M 3}\right) R b_{3} \cos \beta_{b} \\
\vdots \\
\left(k_{N-2}(t) \delta e_{N-2}(M) \zeta_{M N-2}+k_{N-1}(t) \delta e_{N-1}(M) \zeta_{M N-1}\right) R b_{N-1} \cos \beta_{b} \\
k_{N-1}(t) \delta e_{N-1}(M) \zeta_{M N-1} R b_{N} \cos \beta_{b}
\end{array}\right\}
$$

so as to keep a constant total power); (c) a constant resisting torque $C t_{3}=-200$ N.m and finally; (d) a periodic resisting torque $C t_{3}(t)=\left[-200+70 \sin \left(10 \Omega_{1} t\right)\right]$ N.m on pinion 3 , a constant driving torque $C t_{1}=500$ N.m on pinion 1 and a resisting torque on the output gear 4 (so that the total power is constant).

The response curves in Figures 5a and 5c represent the maximum dynamic mesh forces for constant loads and clearly exhibit response peaks which correspond to a natural frequency being excited by the mesh frequency and its harmonics: $5250 \mathrm{rpm} \cong\left(W_{2} / z_{1}\right) \mathrm{rad} . \mathrm{s}^{-1}, 3250 \mathrm{rpm} \cong$ $\left(W_{4} / z_{1}\right) \mathrm{rad} . \mathrm{s}^{-1}, 1600 \mathrm{rpm} \cong\left(W_{4} / z_{1}\right) / 2{\mathrm{rad} . \mathrm{s}^{-1}, 500 \mathrm{rpm}}^{-1}$ $\cong\left(W_{3} / z_{1}\right)$ rad.s ${ }^{-1}$. The results in Figures $5 \mathrm{~b}$ and $5 \mathrm{~d}$ correspond to the cases with time-varying torques (of frequency $10 \Omega_{1}$ ) for which the numerical simulations were performed over at least 5 mesh periods in order to cover the system period $\left(T_{s y s}=\operatorname{LCM}\left(z_{1}=50, f_{t}=10\right) \frac{T_{m}}{x f_{t}}\right.$;
$\left.T_{s y s}=5 T_{m}\right)$. The addition of a periodic torque introduces an additional response peak at $2600 \mathrm{rpm} \cong$ $\left(W_{3} / f_{t}\right)$ rad.s $^{-1}$ on the three stages showing that there exist significant inter-mesh couplings. Moreover, the response curves for (c) and (d) highlight the power circulation influence on dynamic tooth forces since, for the same gear geometry and arrangement, stages 1 and 2 experience higher dynamic loads whereas stage 3 is less loaded compared with the previous examples (a) and (b). Some slight frequency shifts can also be noticed which are probably caused by the changes in nominal tooth loading hence on the mesh stiffness functions (the contact compliance being load dependent). Finally, it should be noticed that the introduction of a periodic component on torque $C t_{1}$ or $C t_{3}$ leads to modified response spectra (not shown here) which exhibit significant modulation side-bands. 
Table 1. Gear data.

\begin{tabular}{|c|c|c|c|c|}
\hline & Pinion 1 & Pinion 2 & Pinion 3 & Pinion 4 \\
\hline Tooth number & 50 & 61 & 48 & 47 \\
\hline Face width $(\mathrm{mm})$ & 14 & 15 & 19 & 15 \\
\hline Module (mm) & \multicolumn{4}{|c|}{2.54} \\
\hline Pressure angle $\left({ }^{\circ}\right)$ & \multicolumn{4}{|c|}{20} \\
\hline Helix angle $\left({ }^{\circ}\right)$ & \multicolumn{4}{|c|}{0 (spur) } \\
\hline Addendum coefficient & \multicolumn{4}{|c|}{1.0} \\
\hline Profile shift coeff. & \multicolumn{4}{|c|}{0.0} \\
\hline Dedendum coefficient & \multicolumn{4}{|c|}{1.4} \\
\hline Relief extent & \multicolumn{4}{|c|}{$\begin{array}{l}28 \% \text { of the active profile } \\
\quad 76 \mu \mathrm{m} \text { for stage } 1\end{array}$} \\
\hline Tip relief amplitude & \multicolumn{4}{|c|}{$70 \mu \mathrm{m}$ for stage 2} \\
\hline & \multicolumn{4}{|c|}{$67 \mu \mathrm{m}$ for stage 3} \\
\hline
\end{tabular}

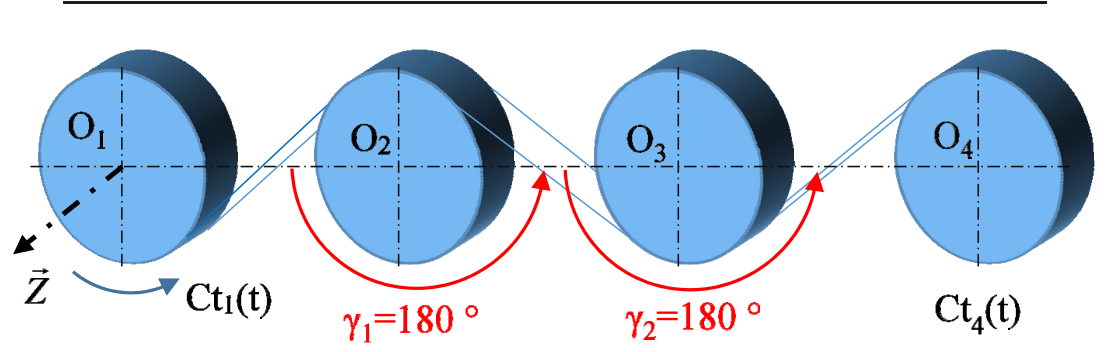

Fig. 4. Three stage idler gear system.

\subsubsection{Linear tooth modifications}

In this section, tip relief was applied on all teeth in order to compensate for the elastic deflections at engagement/end of recess and also improve the tooth load distribution along the path of contact. Tooth reliefs are characterised by an amplitude at tooth tips (depth of modification) and by an extent of modification measured along the path of contact on the base plane. For each stage $m$, the depth of modification depends on the maximum mesh deflection and the extent of relief is expressed as a fraction of the length of the active contact line $\varepsilon_{\alpha_{m}} P b a[11]$.

Based on the results in Figure 6, it can be observed that the system exhibits contact losses between the teeth for some of the reduction stages at some critical speeds mostly at $1600 \mathrm{rpm}, 2600 \mathrm{rpm}$ and $5250 \mathrm{rpm}$. The introduction of short tip reliefs (Table 1) leads to a substantial improvement over the whole speed range (Figs. 6a and $6 \mathrm{~b})$. However, the presence of a periodic torque slightly downgrades the dynamic performance mainly at the additional peaks caused by the time-varying torque excitation around $2600 \mathrm{rpm}$ (Figs. 6c and 6d) for the treated example. This observation confirms that profile modifications can be effective in reducing mesh excitations whereas they hardly modify the contributions from external forcing terms.

\section{Conclusion}

A simplified torsional dynamic model of multi-stage gears has been presented which can account for $N$ stages with any relative orientation of their lines of action. The
Table 2. Tooth critical frequencies.

\begin{tabular}{lc}
\hline & Tooth critical frequency $\left(\right.$ rad.s $\left.^{-1}\right)$ \\
\hline$W_{1}$ & 35937.20 \\
$W_{2}$ & 28614.92 \\
$W_{3}$ & 2792.85 \\
$W_{4}$ & 17101.25 \\
\hline
\end{tabular}

relative phasing between the various mesh excitations is determined analytically and realistic mesh stiffness functions are employed. The results highlight the influence of the power circulation (several power outputs or inputs, etc.) in multi-mesh gear units along with the contributions of external excitations such as periodic torques on dynamic tooth loads and critical speeds. Periodic torques generate additional response peaks and introduce strong modulations in the dynamic response. It has also been shown that short tip profile modifications improve dynamic behaviour over all the range of rotational speeds but have limited influence on external excitation sources. The proposed global (load boundary conditions, power circulation) and local (profile modifications) approach is original as far as the authors know and shed light on multi-mesh gear dynamics. However, torsional models are known to be inaccurate in a number of realistic situations when bearing/casing deflections cannot be ignored. Further research is therefore needed in order to incorporate all three-dimensional displacements particularly when flexible components (casing) can strongly influence the loading conditions on the teeth. Finally, research is currently under way to investigate optimum profile modifications and critically assess the concept of quasistatic transmission errors in multi-stage geared systems. 

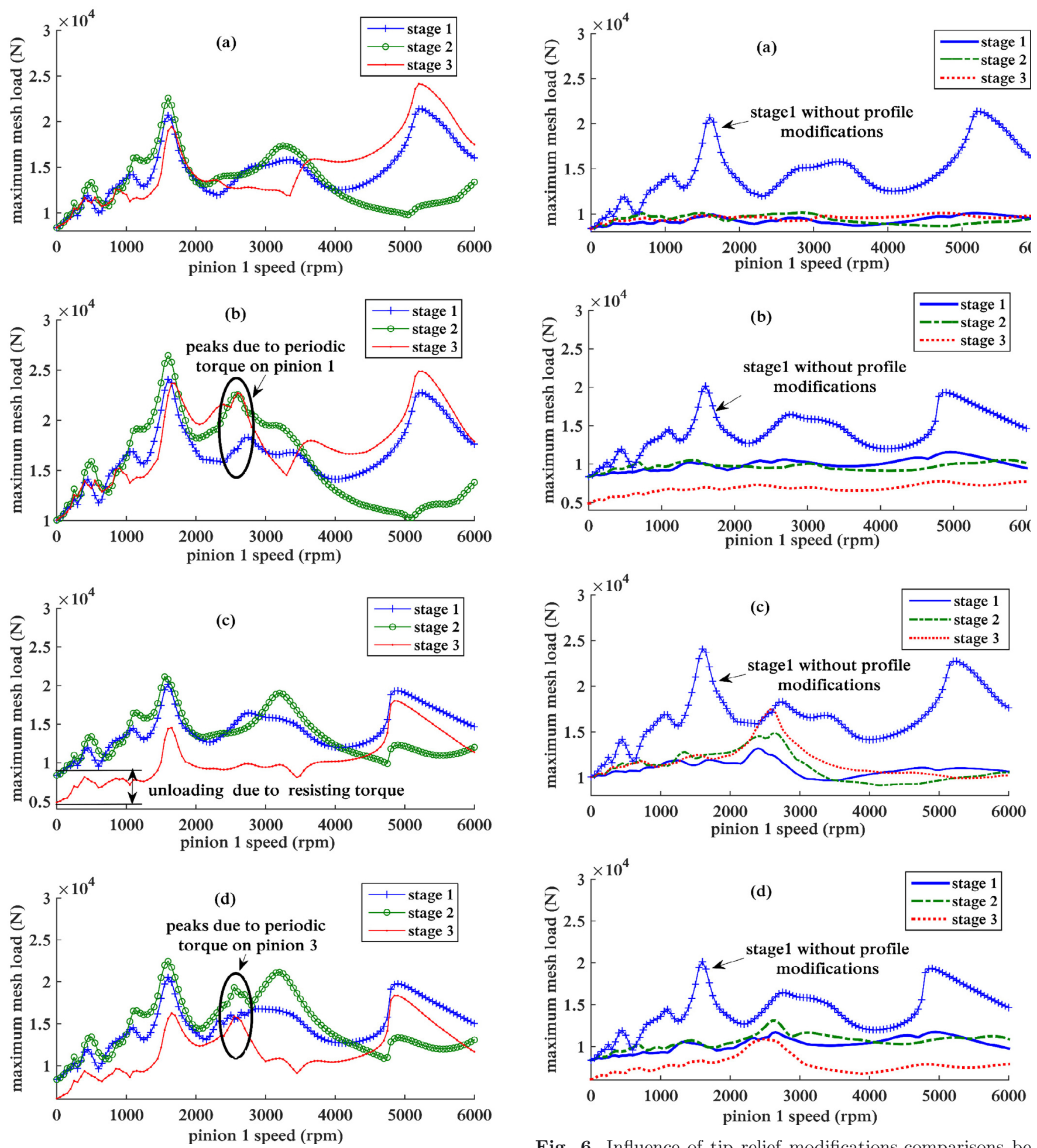

Fig. 5. Influence of (a) constant driving torque on pinion 1, (b) periodic driving torque on pinion 1, (c) constant resisting torque on pinion 3 and (d) periodic resisting torque on pinion 3 on dynamic mesh load.

Fig. 6. Influence of tip relief modifications-comparisons between response curves with and without profile corrections in presence of (a) constant driving torque on pinion 1, (b) constant resisting torque on pinion 3 , (c) periodic driving torque on pinion 1 and $(\mathrm{d})$ periodic resisting torque on pinion 3 . 
Acknowledgements. The authors would like to thank the support of Hispano-Suiza (SAFRAN Group).

\section{References}

[1] F.K. Choy, Y.K. Tu, M. Savage, D.P. Townsend, Vibration signature analysis of multi-stage gear transmission, J. Franklin Institute 328 (1991) 281-298

[2] F.K. Choy, Y.F. Ruan, Y.K. Tu, Modal analysis of multistage gear systems coupled with gearbox vibrations, J. Mech. Des. 114 (1992) 486-497

[3] P. Velex, A. Saada, A model for the dynamic behaviour of multi-stage geared system, Proc. Of the 8th World Congress on Theory of Machines and Mechanisms, Prague, 1991, pp. 621-624

[4] J.P. Raclot, P. Velex, Simulation of the dynamic behaviour of single and multi-stage geared systems with shape deviations and mounting errors by using a spectral method, J. Sound Vib. 220 (1999) 861-903

[5] M. Kubur, A. Kahraman, D. Zini, K. Kienzle, Dynamic analysis of a multi-shaft helical gear transmission by finite elements: Model and experiments, J. Vib. Acous 126 (2004) 398-406

[6] A. Carbonelli, E. Rigaud, J. Perret-Liaudet, E. Pelloli, D. Barday, Robust optimization of a truck timing gear cascade: numerical and experimental results, 21th French Congress of Mechanics, Bordeaux, France, 2013
[7] A. Carbonelli, J. Perret-Liaudet, E. Rigaud, A. Le Bot, Particle swarm optimization as an efficient computational method in order to minimize vibrations of multi-mesh gears transmission, Advances in Acoustics and Vibration, $2011(2011)$

[8] H. Yu, P. Eberhard, Y. Zhao, H. Wang, Sharing behaviour of load transmission on gear pair systems actuated by parallel arrangements of multiple pinions, Mech. Mach. Theory 65 (2013) 58-70

[9] J. Yang, Vibration analysis on multi-mesh gear-trains under combined deterministic and random excitations, Mech. Mach. Theory 59 (2013) 20-33

[10] R.W. Gregory, S.L. Harris, R.G. Munro, Dynamic Behaviour of Spur Gears, Proc. of the Institution Mech. Eng. 178 (1963) 207-218

[11] P. Velex, M. Maatar, A Mathematical Model for Analysing the Influence of Shape Deviations and Mounting Errors on Gear Dynamic Behaviour, J. Sound Vib. 191 (1996) 629-660

[12] J.P. Raclot, Analyse de la réponse dynamique de train d'engrenages à simple et double étage de réduction avec écarts de forme par une méthode spectrale itérative, Thèse, INSA de Lyon, 1997

[13] M. Ajmi, P. Velex, A model for simulating the dynamic behaviour of solid wide-faced spur and helical gear, Mech. Mach. Theory 40 (2005) 173-190

[14] P. Velex, Contribution à l'analyse du comportement dynamique de réducteurs à engrenages à axes parallèles, Thèse, INSA de Lyon, 1988 